\section{Análise espaço-temporal do efeito da poluição do ar na saúde de crianças}

\author{
Space-time analysis of the effect of air pollution \\ on children's health
}

\section{Análisis espacio-temporal del efecto de la contaminación del aire en la salud de los niños}

Emerson Pedreira Matos 1

Valdério Anselmo Reisen 1,2

Faradiba Sarquis Serpa 1,2

Paulo Roberto Prezotti Filho 1,3

Maria de Fátima Silva Leite 1

doi: 10.1590/0102-311X00145418

\section{Correspondência}

E. P. Matos

Universidade Federal do Espirito Santo.

O objetivo foi investigar a associação de curto prazo entre a poluição do ar e atendimentos em emergências por doenças respiratórias, em crianças de 0 a 6 anos. Estudo ecológico, espacial e temporal realizado na Região Metropolitana da Grande Vitória, Espírito Santo, Brasil. Utilizou-se o modelo aditivo generalizado (MAG) de regressão de Poisson, com a variável dependente o número diário de atendimentos por doenças respiratórias, e as variáveis independentes, concentrações diárias dos poluentes atmosféricos $\left(\mathrm{MP}_{10} \mathrm{SO}_{2}, \mathrm{NO}_{2}, \mathrm{O}_{3}\right.$ e CO), temperatura, umidade e precipitação pluviométrica. Por meio das médias diárias das concentrações, foram feitas estimativas para toda a região $e$ análises in loco com a consideração de crianças residentes no entorno de $2 \mathrm{~km}$ de oito estações de monitoramento da qualidade do ar. O incremento de $10 \mu \mathrm{g} / \mathrm{m}^{3}$ nos nivveis de concentração dos poluentes atmosféricos aumentou o risco de atendimento em emergência por doença respiratória. Na região geral, para o $M P_{10}$ o aumento foi de 2,43\%, 2,73\% e 3,29\% nos acumulados de 5, 6 e 7 dias, respectivamente. Para o $\mathrm{SO}_{2}$, o acréscimo foi de 4,47\% no dia da exposição, 5,26\% dois dias após, 6,47\%, 8,8\%, 8,76\% e 7,09\% nos acumulados de 2, 3,4 e 5 dias, respectivamente. O CO apresentou associação significativa para residentes no entorno de duas estações, e o $\mathrm{O}_{3}$ somente em uma. Mesmo dentro dos limites estabelecidos pela Organização Mundial da Saúde, os poluentes $\mathrm{MP}_{10} \mathrm{SO}_{2}, \mathrm{NO}_{2}$ e $\mathrm{O}_{3}$ estão associados ao maior risco para atendimento por doenças respiratórias em crianças de 0 a 6 anos, e alguns efeitos só foram identificados nas localidades desagregadas por região, isto é, in loco, o que possibilita captar maior variabilidade dos dados.

Poluição do Ar; Doenças Respiratórias; Criança
Av. Fernando Ferrari 514, Vitória, ES 29075-073, Brasil. emersonpmatos@gmail.com

1 Universidade Federal do Espírito Santo, Vitória, Brasil. 2 Escola Superior de Ciências, Santa Casa de Misericórdia de Vitória, Vitória, Brasil.

3 Instituto Federal do Espirito Santo, Vitória, Brasil. 


\section{Introdução}

As doenças respiratórias representam a principal causa de morbimortalidade de crianças, e podem ser causadas ou agravadas pela exposição a poluentes atmosféricos emitidos em larga escala em todo o planeta 1,2 . As emissões de material particulado (MP), óxidos de nitrogênio (NOx), compostos orgânicos voláteis (VOCs), dióxido de enxofre $\left(\mathrm{SO}_{2}\right)$ e poluentes fotoquímicos, como o ozônio $\left(\mathrm{O}_{3}\right)$, aumentaram nas últimas décadas devido ao crescimento da frota de veículos automotores e ao crescente processo de industrialização 2. Estudos realizados em grandes centros urbanos comprovam a associação significativa entre os níveis desses poluentes e o número de atendimentos em emergências e hospitalizações por causas respiratórias 3,4,5,6,7,8,9,10,11,12,13,14.

Uma revisão sistemática recente sobre a saúde de crianças brasileiras e o meio ambiente identificou apenas 17 estudos de séries temporais que relacionaram poluição do ar e causas respiratórias, todos realizados na Região Sudeste. Esses estudos mostraram associação positiva entre os níveis dos poluentes e atendimentos em emergências, hospitalizações ou diminuição de função pulmonar 15. Sendo que dois deles foram realizados na Região Metropolitana da Grande Vitória, Espírito Santo 12,16.

A Região Metropolitana da Grande Vitória é predominantemente urbana e altamente industrializada, e em processo de expansão. A qualidade do ar é afetada pela emissão de poluentes provenientes de veículos automotores e de grandes empreendimentos industriais, além do setor de logística, devido à existência de um grande complexo portuário e de aeroporto 17 . As emissões geradas por essas fontes móveis e fixas influenciam diretamente a qualidade do ar da região 17.

Para detectar a associação entre as concentrações de poluentes e causas respiratórias é necessário empregar técnicas estatísticas que permitam isolar os efeitos da poluição do ar, uma vez que podem existir diversos fatores de confusão, como a temperatura e a umidade. Os modelos de séries temporais têm possibilitado análises mais precisas desse tipo de associação.

Em geral, estudos epidemiológicos de séries temporais utilizam uma única estação de monitoramento fixa ou a média das estações para representar a exposição de toda a população aos poluentes. No entanto, essa abordagem pode não refletir a verdadeira exposição das pessoas 18 . Tendo em vista essa situação, uma análise mais realista pode ser feita se for considerada uma pequena área geográfica próxima à estação de monitoramento 19 . Todavia, existe um número limitado de estudos epidemiológicos que usam esse tipo de avaliação, embora existam algumas evidências de que a exposição de erro de classificação em análise de séries temporais tende para um viés das estimativas para baixo e, neste sentido, não limita a importância dos resultados para saúde pública 19 .

Portanto, metodologias nas quais as áreas de abrangência sejam melhor discriminadas espacialmente tornam-se um fator importante para obter inferências mais precisas. Nesse contexto, o objetivo deste estudo foi avaliar, por meio do modelo aditivo generalizado (MAG), a relação de curto prazo entre o número de atendimentos de emergências por problemas respiratórios, em crianças menores de seis anos, com os níveis de poluentes atmosféricos observados na Região Metropolitana da Grande Vitória, levando-se em consideração as variáveis temporais medidas por localidade in loco (área em torno das redes de monitoramento) e por média entre as estações (média regional global).

\section{Métodos}

\section{Área de estudo}

Estudo ecológico, espacial e temporal, realizado na Região Metropolitana da Grande Vitória no período de 1o de janeiro de 2005 a 31 de dezembro de 2010. A Região Metropolitana da Grande Vitória é composta por sete municípios (Vitória, Vila Velha, Cariacica, Serra, Viana, Guarapari e Fundão), abrange uma área de $2.318 .917 \mathrm{~km}^{2}$, com uma população de aproximadamente 1,7 milhão de habitantes, sendo um dos principais polos de desenvolvimento urbano e industrial do Espírito Santo (Instituto Brasileiro de Geografia e Estatística. Censo populacional 2010. https://ww2.ibge.gov.br/home/ estatistica/populacao/censo2010, acessado em 19/Out/2018). As principais atividades poluidoras da região incluem as vias de tráfego, indústrias de diversos seguimentos (siderurgia, pelotização, mineração, cimenteiras), portos, aeroportos, emissões residenciais e comerciais 17 . 
O monitoramento da qualidade do ar na região é realizado por nove estações, que juntas compõem a Rede Automática de Monitoramento da Qualidade do Ar (RAMQAr), gerenciada pelo Instituto Estadual de Meio Ambiente e Recursos Hídricos ${ }^{17}$. Na análise espacial, consideramos a área de influência de cada estação, conforme Santolim ${ }^{20}$. Essa área corresponde a um círculo ao redor da estação, com raio de aproximadamente $2 \mathrm{~km}$. Na época do estudo, oito das nove estações da RAMQAr (Figura 1) estavam em funcionamento.

\section{Figura 1}

Área de abrangência do estudo.
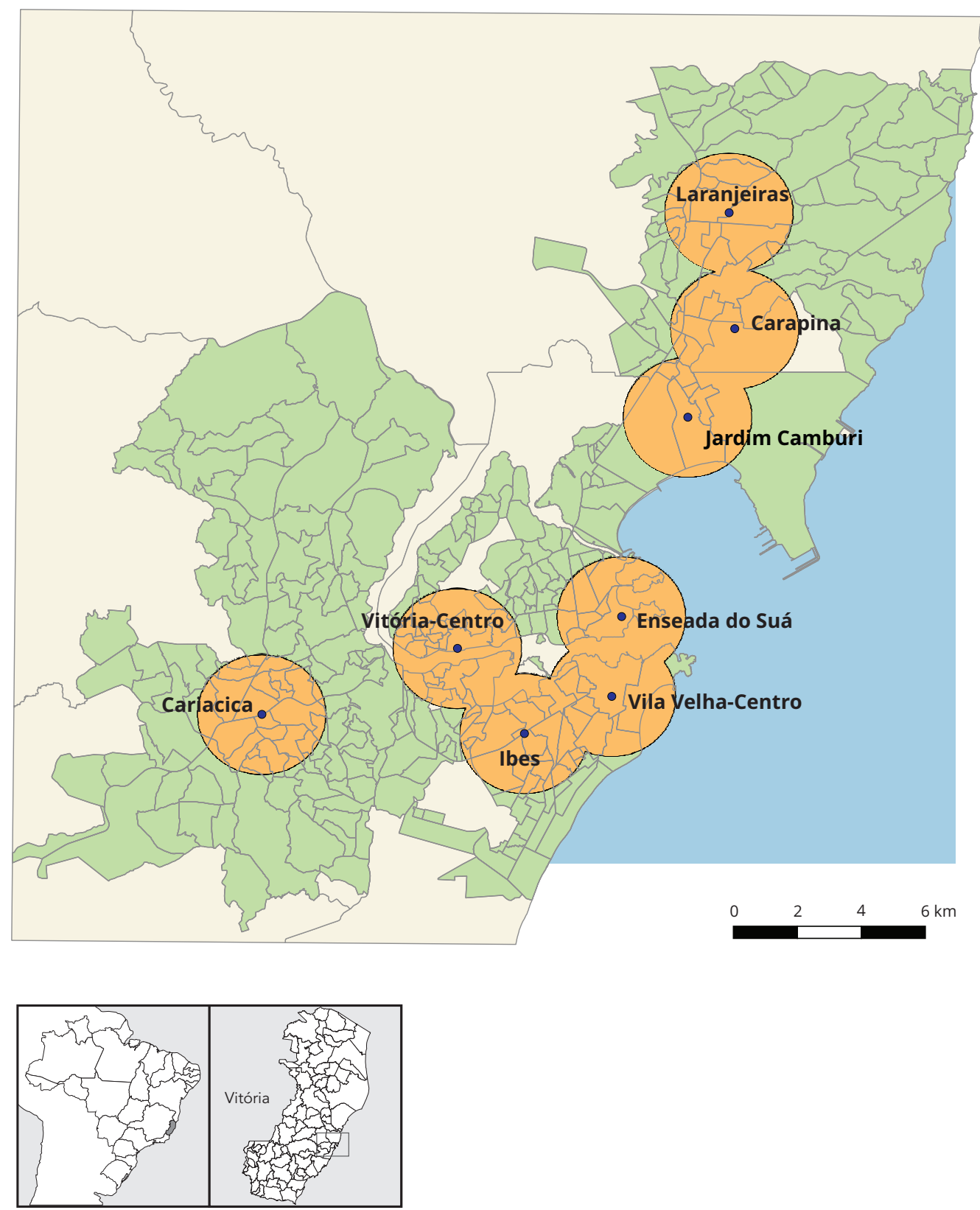


\section{Desfecho de saúde}

Os dados de atendimentos de emergência por causas respiratórias de crianças menores de 6 anos foram obtidos nos setores de emergência de dois hospitais, um da rede pública e outro da rede privada: Hospital Infantil Nossa Senhora da Glória e o Centro Integrado de Atenção à Saúde da Unimed Vitória, respectivamente. As doenças respiratórias foram codificadas de acordo com a 10a revisão da Classificação Internacional de Doenças (CID-10: J00-J99). Selecionamos os atendimentos de crianças que residiam nos bairros localizados na área de influência de cada uma das estações da RAMQAr.

\section{Poluentes ambientais e variáveis meteorológicas}

As concentrações de material particulado com volume aerodinâmico de até 10 micrômetros $\left(\mathrm{MP}_{10}\right)$, $\mathrm{SO}_{2}$, dióxido de nitrogênio $\left(\mathrm{NO}_{2}\right), \mathrm{O}_{3}$ e monóxido de carbono $(\mathrm{CO})$ para o período foram cedidas pelo Instituto Estadual de Meio Ambiente e Recursos Hídricos (IEMA), que é o responsável pelas nove estações da RAMQAr: Laranjeiras, Carapina, Cidade Continental, Jardim Camburi, Enseada do Suá, Vitória-Centro, Ibes, Vila Velha-Centro e Cariacica. Essas estações automáticas coletam e analisam continuamente amostras do ar e processam essas informações na forma de médias horárias, no próprio local e em tempo real. A metodologia de medição do $\mathrm{MP}_{10}$ é a Tapered Element Oscillating Microbalance (TEOM), que afere de forma contínua a concentração de massa. Para medir o $\mathrm{SO}_{2}$ é aplicado o princípio da fluorescência dos raios ultravioleta. Para o $\mathrm{NO}_{2}$ é utilizada uma combinação do tipo de modulação de fluxo cruzado duplo, também usado na medição do $\mathrm{CO}$ e do $\mathrm{O}_{3}$, com o princípio de quimiluminescência com o método de cálculo diferencial ${ }^{17}$. Sendo assim, calculamos as médias diárias dos poluentes $\mathrm{MP}_{10}$ e $\mathrm{SO}_{2}$, e utilizamos a concentração máxima horária de $\mathrm{NO}_{2} \mathrm{e}$ a concentração máxima diária das médias móveis de oito horas de $\mathrm{CO}$ e $\mathrm{O}_{3}$. Todos os poluentes em todas as estações foram medidos em micrograma por metro cúbico $\left(\mu \mathrm{g} / \mathrm{m}^{3}\right)$.

As variáveis meteorológicas consideradas foram: a temperatura expressa em graus Celsius $\left({ }^{\circ} \mathrm{C}\right)$; a umidade relativa do ar expressa em porcentagens (\%), registradas pelos monitores de Carapina e Cariacica; e a precipitação pluviométrica expressa em milímetros ( $\mathrm{mm})$, registrada no monitor de Carapina, que são as estações que medem estes parâmetros. Com essas informações, calculamos as médias aritméticas das medidas de umidade e temperatura (máxima, média e mínima) registradas e a precipitação pluviométrica representada pelo volume diário de chuva. Incluímos também, para cada dia, os valores de temperatura e umidade do dia corrente do atendimento médico (lag0), do dia anterior (lag1), de dois dias anteriores (lag2) e médias móveis de dois e três dias anteriores (mm01, mm02).

\section{Dados faltantes}

As falhas no monitoramento dos poluentes na RAMQAr ocorridas durante o período estudado, tanto em dias isolados como em dias consecutivos, causaram lacunas nos registros das concentrações e foram corrigidas pelo método de imputação, seguindo metodologia descrita por Junger 21. Nesse método, as estimativas obtidas são explicadas pela correlação espacial entre os diferentes níveis do mesmo poluente nos diferentes monitores e pela autocorrelação dos níveis do poluente no mesmo monitor, ao longo do tempo. Os padrões temporais são modelados por meio um modelo gaussiano autoregressivo integrado e de médias móveis, um ARIMA. Esse algoritmo é especialmente adaptado para dados climáticos com medições ausentes de alguns monitores ao longo de uma determinada região. Os limites superior e inferior de imputação dos dados faltantes foram as máximas e mínimas concentrações, respectivamente, observadas na série histórica das concentrações de cada poluente.

\section{Análise estatística}

A estratégia de modelagem consistiu em definir um modelo central com todas as informações conhecidas (tendência, sazonalidade, dias da semana, feriados e as condições meteorológicas), a fim de explicar a variabilidade do número de atendimentos por doenças respiratórias, exceto a concentração dos poluentes. 
A escolha das variáveis e covariáveis para compor o modelo foi baseada em testes e diagnósticos em cada etapa do processo de modelagem. Os diagnósticos foram baseados na análise residual e no critério Akaike (AIC) 22.

\section{Modelo aditivo generalizado}

O número diário de atendimentos médicos representa um processo de contagem e o modelo aditivo generalizado (MAG), com distribuição marginal de Poisson, foi a ferramenta estatística utilizada para estimar a forma da curva da relação entre desfecho de saúde e poluição do ar 5,13,14.

Seja $\left\{Y_{t}\right\} \equiv\left\{Y_{t}\right\}_{t} Z$ uma série de contagem, ou seja, $y_{t} \in\{0,1, \ldots\}$. A distribuição condicional de $Y_{t}$, dado o passado $\mathrm{F}_{\mathrm{t}-1}$ que contém toda informação disponível até o momento $\mathrm{t}$ - 1, é denotada por:

$$
p\left(y_{\mathrm{t}}, \mu_{t} \mid F_{t-1}\right)=\frac{e^{-\mu_{t}} \mu_{t}^{y_{t}}}{y_{\mathrm{t} !}}
$$

em que $\mu_{t}$ representa o valor esperado (média) de $Y_{t}$. Assim, dada uma amostra $Y, \ldots, Y_{n}$, composta de " $\mathrm{n}$ " variáveis aleatórias mutuamente condicionalmente independentes, pertencentes à " $\mathrm{Y}_{\mathrm{t}}$ ", a função de log-verossimilhança condicional é dada por:

$$
l(\mu)=\sum_{i=1}^{n} \ln p\left(y_{t} ; \mu_{t} \mid F_{t-1}\right) \propto \sum_{i=1}^{n}\left(Y_{t} \ln \mu_{t}-\mu_{t}\right)
$$

em que o vetor $\mu=\left(\mu 1, \ldots, \mu_{n}\right)$ depende dos parâmetros e do processo $\left\{Y_{t}\right\}$. Seja $X_{t}=\left[X_{1 t}, \ldots, X_{p t}\right]^{T}$ o vetor de covariáveis de dimensão $\mathrm{p}$ no tempo $\mathrm{t}$, em que $\mathrm{T}$ denota a transposta, que pode incluir valores passados de $\mathrm{Y}_{\mathrm{t}}$ e outras informações auxiliares, tais como poluentes e variáveis de confusão (tendência, sazonalidade e variáveis meteorológicas, entre outros). Neste estudo, a sequência $\mathrm{X}_{1 \mathrm{t}}, \ldots, \mathrm{X}_{\mathrm{qt}}$ denota as concentrações dos poluentes $\mathrm{MP}_{10}, \mathrm{SO}_{2}, \mathrm{NO}_{2}, \mathrm{O}_{3}$ e $\mathrm{CO}$, portanto, $\mathrm{q}=5$, e $\mathrm{X}_{(\mathrm{q}+1) t}, \ldots, \mathrm{X}_{\mathrm{pt}}$ indica as variáveis de confusão no tempo $\mathrm{t},(\mathrm{p}>\mathrm{q})$.

A relação entre o vetor é dada por:

$$
\ln \left(\mu_{t}\right)=\sum_{j=0}^{q} \beta_{j} X_{j t}+\sum_{j=q+1}^{p} f_{j}\left(X_{j t}\right)
$$

em que $\left(\beta_{0}, \beta\right), \operatorname{com} \beta=\left(\beta 1, \ldots, \beta_{\mathrm{q}}\right)^{\mathrm{T}}$ é o vetor dos coeficientes a serem estimados ( $\beta_{\mathrm{j}}$ é o coeficiente j-ésima covariável) e fj é a função suavisadora para a j-ésima variável de confusão. Além disso, $\beta_{0}$ indica o intercepto da curva e está associado a $\mathrm{X}_{0 \mathrm{t}}=1$ para todo t. Todo o processo de modelagem foi realizado no software R (http://www.r-project.org) com o pacote ARES 23.

O risco relativo (RR) de uma covariável poluente Xi, $\mathrm{j}=1, \ldots, \mathrm{q}$ é dado como sendo a variação relativa na contagem esperada de eventos de doenças respiratórias pela variação $\xi$ de unidade na covariável enquanto mantidas as outras covariáveis fixas. De acordo com Baxter et al. ${ }^{24}$, fórmula (8), o RR é dado por:

$$
R R_{X_{j}}(\xi)=\frac{E\left(Y \mid X_{j}=\xi, X_{i}, i \neq j\right)}{E\left(Y \mid X_{j}=0, X_{i}, i \neq j\right)}
$$

Para a regressão de Poisson, o RR não depende dos valores $\mathrm{x}_{\mathrm{i}}, \mathrm{i} \neq \mathrm{j}$ das outras covariáveis e pode ser expresso como:

$$
R R_{X_{j}}(\xi)=\exp \left(\beta_{j} \xi\right)
$$

Para o modelo MAG com distribuição marginal de Poisson, o RR e o seu intervalo de confiança (IC) aproximado, em um nível de significância $\alpha$, de uma covariável $X_{\mathfrak{j}}, j=1 \ldots q$, é estimado da seguinte forma:

$$
\begin{gathered}
R R_{X_{j}}(\xi)_{X_{j}}=\exp \left(\hat{\beta}_{J} \xi\right) \\
I C\left(R R_{X_{j}}(\xi)\right)=\exp \left(\hat{\beta}_{J} \xi \pm z_{\alpha / 2}\left(\hat{\beta}_{J}\right) \xi\right)
\end{gathered}
$$


$\hat{\beta}_{J}$ é o coeficiente estimado associado ao poluente $\mathrm{Xj}$ em um estudo com erro padrão $\mathrm{s}\left(\hat{\beta}_{J}\right)$ e $z_{\alpha / 2}$ é o quantil $\alpha / 2$ da distribuição normal padrão. Num nível de significância $\alpha$, a hipótese a ser testada é definida como $H_{0}: R_{x j}=1$ contra $H_{0}: R_{x j}>1$, em que $R_{x j}=R_{x j}(1)$, ou seja, $R R$ da variação da unidade em $\mathrm{X}_{\mathrm{j}}$. A rejeição de $\mathrm{H}_{0}$ implica estatisticamente que o respectivo poluente tem um efeito adverso significativo na saúde.

Neste estudo, os cálculos dos valores dos RR(xj) correspondem ao aumento de $1.000 \mu \mathrm{g} / \mathrm{m}^{3}$ nos níveis de $\mathrm{CO}$ e de $10 \mu \mathrm{g} / \mathrm{m}^{3}$ para os demais poluentes. Os resultados são apresentados em aumentos percentuais nos números de atendimentos médicos e são calculados por meio da expressão:

$$
\% R R(x)=(R R(x)-1) x 100
$$

\section{Defasagem (lag)}

As manifestações biológicas dos efeitos da poluição na saúde humana, aparentemente, apresentam um comportamento que mostra uma defasagem em relação à exposição do indivíduo aos agentes poluidores, ou seja, eventos que ocorrem num determinado dia podem estar associados aos níveis de poluição daquele dia e/ou de dias anteriores. Por isso, de acordo com estudos anteriores 25,26, decidimos investigar a associação entre o número de atendimentos por doença respiratória e os níveis de poluição no dia do atendimento na emergência (lag0) e nos dias anteriores (lag1, lag2, lag3). O efeito acumulado foi avaliado com as médias móveis de dois a oito dias (MA01, MA02, MA03, MA04, MA05, MA06, MA07) 27,28.

O projeto foi aprovado no Comitê de Ética Profissional (CEP) do Centro de Ciência da Saúde, Universidade Federal do Espírito Santo, sob o no 04/11, em 14 de maio de 2011.

\section{Resultados}

No período estudado foram registrados 46.421 atendimentos em emergência por doenças respiratórias em crianças de 0 a 6 anos, residentes nas áreas de abrangência de oito estações de monitoramento da RAMQAr. A média diária de atendimentos na Região Metropolitana da Grande Vitória foi de 21,19 $(\mathrm{DP}=9,90)$ e variou de 1,72 a 4,84 atendimentos/dia, com maiores médias mensais entre os meses de março a junho, outono e início do inverno. O maior número de atendimentos foi de crianças residentes na região da Enseada do Suá.

A temperatura média no período variou de 20,85 a $29,36^{\circ} \mathrm{C}$, a quantidade de chuva variou de $0 \mathrm{~mm}$ a $117,80 \mathrm{~mm}$ (média $=3,78 \mathrm{~mm}$ ) e a umidade relativa do ar variou de $61,79 \%$ a $97,27 \%$ (média $=77,47 \%$ ).

As concentrações dos poluentes não apresentaram comportamento uniforme entre as diferentes estações da RAMQAr. As concentrações médias mais altas registradas no período foram: $\mathrm{MP}_{10}$ $\left(43,06 \mu \mathrm{g} / \mathrm{m}^{3}\right)$ na região de Cariacica, $\mathrm{SO}_{2}\left(16,32 \mu \mathrm{g} / \mathrm{m}^{3}\right)$ na Enseada do Suá, $\mathrm{O}_{3}\left(38,66 \mu \mathrm{g} / \mathrm{m}^{3}\right) \mathrm{em}$ Ibes e $\mathrm{NO}_{2}\left(44,10 \mu \mathrm{g} / \mathrm{m}^{3}\right)$ e $\mathrm{CO}\left(1.730,91 \mu \mathrm{g} / \mathrm{m}^{3}\right)$ em Vitória-Centro. Na Tabela 1 são apresentadas as concentrações mínimas, médias e máximas, assim como os quartis $25 \%$, 50\% e $75 \%$ e o desvio padrão (DP) dos poluentes.

A série de contagens diárias de atendimentos, na região geral, foi suavizada por uma "spline" com 12 graus de liberdade, definida por meio do critério de modelagem AIC e da análise residual. $\mathrm{O}$ ajuste não paramétrico evidenciou sazonalidade e uma tendência decrescente ao longo do tempo, fatores de confusão que foram incluídos no processo de modelagem. O período do outono (março) para o inverno (junho) evidenciou uma sazonalidade com aumento do número de atendimentos por causas respiratórias.

A análise do diagnóstico do ajuste do modelo, descrito anteriormente, por meio dos resultados obtidos da regressão de Poisson para a estimação do efeito do $\mathrm{MP}_{10}$ da média móvel de seis dias para Região Metropolitana da Grande Vitória, está apresentada na Figura 2.

$\mathrm{O}$ coeficiente estimado para o $\mathrm{MP}_{10}$ foi igual a 0,0032 , com um erro padrão de 0,0015 e valor de $\mathrm{p}$ de 0,0301, o qual representamos na forma [MM6, Região Metropolitana da Grande Vitória] (0,0032; $0,00145 ; 0,0301)$. 
Tabela 1

Estatística descritiva dos atendimentos por doenças respiratórias, das médias diárias do $\mathrm{MP}_{10}\left(\mu \mathrm{g} / \mathrm{m}^{3}\right), \mathrm{SO}_{2}\left(\mu \mathrm{g} / \mathrm{m}^{3}\right)$ e $\mathrm{NO}_{2}\left(\mu \mathrm{g} / \mathrm{m}^{3}\right)$ e das médias móveis de 8 horas do $\mathrm{O}_{3}\left(\mu \mathrm{g} / \mathrm{m}^{3}\right)$ e $\mathrm{CO}\left(\mu \mathrm{g} / \mathrm{m}^{3}\right)$, em cada Rede Automática de Monitoramento da Qualidade do $\operatorname{Ar}$ (RAMQAr), 2005-2010.

\begin{tabular}{|c|c|c|c|c|c|c|c|}
\hline & Média & DP & Mínimo & Máximo & p25 & Mediana & p75 \\
\hline \multicolumn{8}{|l|}{ Carapina } \\
\hline ADR & 2,31 & 2,10 & 0 & 17 & 1 & 2 & 3 \\
\hline $\mathrm{MP}_{10}$ & 23,02 & 7,96 & 5,75 & 88,25 & 18,08 & 21,67 & 26,5 \\
\hline $\mathrm{SO}_{2}$ & - & - & - & - & - & - & - \\
\hline Tmpmin & 20,85 & 2,47 & 13,10 & 25,98 & 19,05 & 21,15 & 22,80 \\
\hline Tmpmed & 24,43 & 2,45 & 17,00 & 30,80 & 22,63 & 24,41 & 26,36 \\
\hline Tmpmax & 29,36 & 3,28 & 19,40 & 39,70 & 27,20 & 29,43 & 31,60 \\
\hline Umidade & 77,47 & 6,21 & 61,79 & 97,27 & 73,11 & 77,10 & 81,44 \\
\hline Chuva & 3,78 & 11,31 & 0,00 & 117,80 & 0,00 & 0,00 & 1,80 \\
\hline \multicolumn{8}{|l|}{ Vila Velha-Centro } \\
\hline ADR & 2,25 & 1,76 & 0 & 12 & 1 & 2 & 3 \\
\hline $\mathrm{MP}_{10}$ & 23,49 & 8,22 & 5,04 & 90,75 & 18,04 & 22,33 & 27,48 \\
\hline $\mathrm{SO}_{2}$ & 11,99 & 5,80 & 0,50 & 54,16 & 8,61 & 11,21 & 13,98 \\
\hline Tmpmin & 20,85 & 2,47 & 13,1 & 25,98 & 19,05 & 21,15 & 22,80 \\
\hline Tmpmed & 24,43 & 2,45 & 17,00 & 30,80 & 22,63 & 24,41 & 26,36 \\
\hline Tmpmax & 29,36 & 3,28 & 19,40 & 39,70 & 27,20 & 29,43 & 31,60 \\
\hline Umidade & 77,47 & 6,21 & 61,79 & 97,27 & 73,11 & 77,10 & 81,44 \\
\hline Chuva & 3,78 & 11,31 & 0,00 & 117,80 & 0,00 & 0,00 & 1,80 \\
\hline \multicolumn{8}{|l|}{ Cariacica } \\
\hline ADR & 2,28 & 1,91 & 0 & 13 & 1 & 2 & 3 \\
\hline $\mathrm{MP}_{10}$ & 43,06 & 15,94 & 8,83 & 117,88 & 32,52 & 41,13 & 51,46 \\
\hline $\mathrm{NO}_{2}$ & 28,06 & 11,18 & 4,01 & 97,64 & 19,28 & 26,79 & 35,78 \\
\hline $\mathrm{NO}_{2}$ (máximo) & 50,65 & 19,35 & 8,65 & 220,25 & 36,8 & 49,20 & 62,36 \\
\hline $\mathrm{SO}_{2}$ & 5,5 & 2,62 & 0,00 & 19,82 & 3,42 & 5,32 & 6,98 \\
\hline $\mathrm{CO}$ & 609,79 & 266,86 & 87,28 & 3700,05 & 412,67 & 577,28 & 769,46 \\
\hline $\mathrm{O}_{3}$ & 24,78 & 9,32 & 4,50 & 73,35 & 18,33 & 23,76 & 30,45 \\
\hline $\mathrm{O}_{3}$ (máximo) & 47,9 & 13,93 & 12,08 & 120,00 & 37,93 & 46,94 & 56,40 \\
\hline $\mathrm{O}_{3}(8 \mathrm{~h})$ & 37,79 & 12,59 & 8,54 & 105,69 & 28,31 & 37,30 & 46,02 \\
\hline Tmpmin & 20,85 & 2,47 & 13,10 & 25,98 & 19,05 & 21,15 & 22,8 \\
\hline Tmpmed & 24,43 & 2,45 & 17,00 & 30,80 & 22,63 & 24,41 & 26,36 \\
\hline Tmpmax & 29,36 & 3,28 & 19,4 & 39,70 & 27,2 & 29,43 & 31,60 \\
\hline Umidade & 77,47 & 6,21 & 61,79 & 97,27 & 73,11 & 77,10 & 81,44 \\
\hline Chuva & 3,78 & 11,31 & 0,00 & 117,80 & 0,00 & 0,00 & 1,80 \\
\hline \multicolumn{8}{|l|}{ Enseada do Suá } \\
\hline ADR & 4,84 & 2,86 & 0 & 19 & 3 & 4 & 6 \\
\hline $\mathrm{MP}_{10}$ & 29,39 & 9,18 & 7,46 & 83,58 & 23,21 & 28,13 & 34,46 \\
\hline $\mathrm{NO}_{2}$ & 23,69 & 8,00 & 5,23 & 81,40 & 18,06 & 22,75 & 28,35 \\
\hline $\mathrm{NO}_{2}$ (máximo) & 44,1 & 14,29 & 9,04 & 198,8 & 35,51 & 42,90 & 51,40 \\
\hline $\mathrm{SO}_{2}$ & 16,32 & 7,91 & 1,92 & 49,81 & 10,49 & 15,02 & 21,99 \\
\hline $\mathrm{CO}$ & 783,3 & 276,71 & 80,22 & 2250,68 & 580,37 & 740,89 & 944,23 \\
\hline $\mathrm{O}_{3}$ & 29,91 & 8,84 & 9,08 & 69,52 & 24,51 & 28,51 & 34,27 \\
\hline $\mathrm{O}_{3}$ (máximo) & 49,05 & 13,12 & 10,18 & 115,03 & 40,30 & 46,81 & 56,08 \\
\hline $\mathrm{O}_{3}(8 \mathrm{~h})$ & 38,66 & 10,99 & 9,40 & 94,62 & 31,45 & 36,72 & 44,79 \\
\hline Tmpmin & 20,85 & 2,47 & 13,1 & 25,98 & 19,05 & 21,15 & 22,8 \\
\hline Tmpmed & 24,43 & 2,45 & 17,00 & 30,80 & 22,63 & 24,41 & 26,36 \\
\hline Tmpmax & 29,36 & 3,28 & 19,40 & 39,70 & 27,20 & 29,43 & 31,60 \\
\hline Umidade & 77,47 & 6,21 & 61,79 & 97,27 & 73,11 & 77,10 & 81,44 \\
\hline Chuva & 3,78 & 11,31 & 0,00 & 117,80 & 0,00 & 0,00 & 1,80 \\
\hline
\end{tabular}

(continua) 


\begin{tabular}{|c|c|c|c|c|c|c|c|}
\hline & Média & DP & Mínimo & Máximo & p25 & Mediana & p75 \\
\hline \multicolumn{8}{|l|}{ Laranjeiras } \\
\hline ADR & 2,05 & 1,83 & 0 & 12 & 1 & 2 & 3 \\
\hline $\mathrm{MP}_{10}$ & 32,9 & 11,39 & 7,45 & 106,88 & 25,21 & 31,6 & 38,86 \\
\hline $\mathrm{NO}_{2}$ & 22,07 & 7,17 & 3,36 & 59,38 & 17,12 & 21,16 & 25,73 \\
\hline $\mathrm{NO}_{2}$ (máximo) & 41,23 & 14,85 & 10,00 & 115,27 & 31,19 & 38,82 & 47,70 \\
\hline $\mathrm{SO}_{2}$ & 12,61 & 5,79 & 2,07 & 52,61 & 8,59 & 11,61 & 15,93 \\
\hline $\mathrm{CO}$ & 647,58 & 175,55 & 212,01 & 1750,02 & 529,6 & 619,98 & 724,33 \\
\hline $\mathrm{O} 3$ & 32,58 & 9,05 & 10,26 & 74,18 & 26,54 & 30,90 & 37,05 \\
\hline $\mathrm{O}_{3}$ (máximo) & 52,46 & 16,34 & 18,15 & 139,43 & 41,26 & 48,89 & 60,26 \\
\hline $\mathrm{O}_{3}(8 \mathrm{~h})$ & 43,34 & 12,98 & 14,34 & 106,38 & 34,60 & 40,55 & 50,01 \\
\hline Tmpmin & 20,85 & 2,47 & 13,10 & 25,98 & 19,05 & 21,15 & 22,8 \\
\hline Tmpmed & 24,43 & 2,45 & 17,00 & 30,80 & 22,63 & 24,41 & 26,36 \\
\hline Tmpmax & 29,36 & 3,28 & 19,40 & 39,70 & 27,20 & 29,43 & 31,60 \\
\hline Umidade & 77,47 & 6,21 & 61,79 & 97,27 & 73,11 & 77,10 & 81,44 \\
\hline Chuva & 3,78 & 11,31 & 0,00 & 117,80 & 0,00 & 0,00 & 1,80 \\
\hline \multicolumn{8}{|l|}{ Ibes } \\
\hline ADR & 1,72 & 1,48 & 0 & 9 & 1 & 1 & 3 \\
\hline $\mathrm{MP}_{10}$ & 29,24 & 9,67 & 5,00 & 88,13 & 23,19 & 28,13 & 34,46 \\
\hline $\mathrm{NO}_{2}$ & 20,32 & 5,98 & 4,80 & 58,03 & 16,4 & 19,69 & 23,89 \\
\hline $\mathrm{NO}_{2}$ (máximo) & 38,39 & 11,15 & 9,60 & 100,5 & 31 & 37,70 & 45,99 \\
\hline $\mathrm{SO}_{2}$ & 10,73 & 6,20 & 0,25 & 41,38 & 5,97 & 9,79 & 14,15 \\
\hline $\mathrm{CO}$ & 657,24 & 267,07 & 155,4 & 2551,97 & 475,72 & 608,19 & 773,41 \\
\hline $\mathrm{O}_{3}$ & 40,15 & 12,23 & 13,29 & 102,33 & 31,36 & 37,87 & 47,19 \\
\hline $\mathrm{O}_{3}$ (máximo) & 65,78 & 17,09 & 22,20 & 148,00 & 53,88 & 64,01 & 75,60 \\
\hline $\mathrm{O}_{3}(8 \mathrm{~h})$ & 54,48 & 16,38 & 17,89 & 140,11 & 42,34 & 52,37 & 64,51 \\
\hline Tmpmin & 20,85 & 2,47 & 13,10 & 25,98 & 19,05 & 21,15 & 22,80 \\
\hline Tmpmed & 24,43 & 2,45 & 17,00 & 30,80 & 22,63 & 24,41 & 26,36 \\
\hline Tmpmax & 29,36 & 3,28 & 19,40 & 39,70 & 27,2 & 29,43 & 31,60 \\
\hline Umidade & 77,47 & 6,21 & 61,79 & 97,27 & 73,11 & 77,10 & 81,44 \\
\hline Chuva & 3,78 & 11,31 & 0,00 & 117,80 & 0,00 & 0,00 & 1,80 \\
\hline \multicolumn{8}{|l|}{ Jardim Camburi } \\
\hline $\mathrm{ADR}$ & 2,24 & 1,82 & 0 & 12 & 1 & 2 & 3 \\
\hline $\mathrm{MP}_{10}$ & 26,95 & 8,06 & 5,46 & 78,08 & 21,50 & 25,92 & 31,63 \\
\hline $\mathrm{NO}_{2}$ & 24,91 & 7,85 & 0,92 & 56,83 & 19,06 & 24,02 & 30,17 \\
\hline $\mathrm{NO}_{2}$ (máximo) & 42,07 & 12,76 & 1,17 & 127,04 & 32,5 & 40,94 & 49,98 \\
\hline $\mathrm{SO}_{2}$ & 14,15 & 7,49 & 0,96 & 54,70 & 9,73 & 12,35 & 16,12 \\
\hline \multicolumn{8}{|l|}{$\mathrm{CO}$} \\
\hline Tmpmin & 20,85 & 2,47 & 13,10 & 25,98 & 19,05 & 21,15 & 22,8 \\
\hline Tmpmed & 24,43 & 2,45 & 17,00 & 30,8 & 22,63 & 24,41 & 26,36 \\
\hline Tmpmax & 29,36 & 3,28 & 19,40 & 39,7 & 27,2 & 29,43 & 31,6 \\
\hline Umidade & 77,47 & 6,21 & 61,79 & 97,27 & 73,11 & 77,10 & 81,44 \\
\hline Chuva & 3,78 & 11,31 & 0,00 & 117,8 & 0,00 & 0,00 & 1,80 \\
\hline
\end{tabular}

(continua) 


\begin{tabular}{|c|c|c|c|c|c|c|c|}
\hline & Média & DP & Mínimo & Máximo & p25 & Mediana & p75 \\
\hline \multicolumn{8}{|l|}{ Vitória-Centro } \\
\hline ADR & 3,5 & 2,39 & 0 & 15 & 2 & 3 & 5 \\
\hline $\mathrm{MP}_{10}$ & 26,09 & 7,23 & 6,08 & 70,42 & 21,51 & 25,08 & 30,08 \\
\hline $\mathrm{NO}_{2}$ & 29,87 & 10,24 & 6,56 & 109,68 & 22,56 & 28,77 & 36,28 \\
\hline $\mathrm{NO}_{2}$ (máximo) & 55,78 & 15,27 & 13,1 & 168,35 & 46,38 & 54,71 & 63,83 \\
\hline $\mathrm{SO}_{2}$ & 15,77 & 6,32 & 2,62 & 44,63 & 10,85 & 15,29 & 19,85 \\
\hline $\mathrm{CO}$ & 1730,9 & 715,3 & 451,73 & 4814,2 & 1201,6 & 1611,22 & $2.152,20$ \\
\hline Tmpmin & 20,85 & 2,47 & 13,10 & 25,98 & 19,05 & 21,15 & 22,8 \\
\hline Tmpmed & 24,43 & 2,45 & 17,00 & 30,80 & 22,63 & 24,41 & 26,36 \\
\hline Tmpmax & 29,36 & 3,28 & 19,40 & 39,70 & 27,20 & 29,43 & 31,6 \\
\hline Umidade & 77,47 & 6,21 & 61,79 & 97,27 & 73,11 & 77,10 & 81,44 \\
\hline Chuva & 3,78 & 11,31 & 0,00 & 117,80 & 0,00 & 0,00 & 1,80 \\
\hline \multicolumn{8}{|c|}{ Região Metropolitana da Grande Vitória } \\
\hline ADR & 21,19 & 9,9 & 2 & 64 & 14 & 20 & 26 \\
\hline $\mathrm{MP}_{10}$ & 29,27 & 7,73 & 7,86 & 75,9 & 24,42 & 28,66 & 33,59 \\
\hline $\mathrm{NO}_{2}$ (máximo) & 45,37 & 11,05 & 19,06 & 114,93 & 37,31 & 44,47 & 52,26 \\
\hline $\mathrm{SO}_{2}$ & 12,44 & 3,11 & 4,89 & 26,48 & 10,07 & 12,16 & 14,57 \\
\hline $\mathrm{CO}$ & 885,76 & 231,24 & 295,17 & 2141,53 & 724,83 & 866,60 & 1031,10 \\
\hline $\mathrm{O}_{3}(8 \mathrm{~h})$ & 43,57 & 10,83 & 16,93 & 93,63 & 35,54 & 42,21 & 49,91 \\
\hline Tmpmin & 20,85 & 2,47 & 13,10 & 25,98 & 19,05 & 21,15 & 22,80 \\
\hline Tmpmed & 24,43 & 2,45 & 17,00 & 30,80 & 22,63 & 24,41 & 26,36 \\
\hline Tmpmax & 29,36 & 3,28 & 19,40 & 39,70 & 27,20 & 29,43 & 31,60 \\
\hline Umidade & 77,47 & 6,21 & 61,79 & 97,27 & 73,11 & 77,10 & 81,44 \\
\hline Chuva & 3,78 & 11,31 & 0,00 & 117,80 & 0,00 & 0,00 & 1,80 \\
\hline
\end{tabular}

ADR: número diário de atendimentos por doenças respiratórias; DP: desvio padrão; Tmpmin: temperatura mínima diária; Tmpmed: temperatura média diária; Tmpmax: temperatura máxima diária.

Nota: "umidade" representa o índice diário de umidade relativa do ar e "chuva" é o índice pluviométrico diário.

O ajuste do modelo aos dados relativos às áreas in loco resultaram [MM6, Vilha Velha-Centro] (0,0086; 0,0036; 0.0173), [MM6, Jardim Camburi] (0,0114; 0,0045; 0,0112), [MM6, Cariacica] (0,0044, 0,0021, 0,0331), [MM6, Carapina] $(0,0116 ; 0,0043 ; 0,0074)$. Os resultados relativos às outras localidades não foram estatisticamente significativos.

Com os resultados dos valores estimados pelos modelos calculamos os percentuais de risco relativo $(\mathrm{RR})$ correspondentes à estimativa e procedemos às análises apresentadas.

Observamos que não houve evidência empírica de mal ajuste do modelo, isto é, os resíduos não são correlacionados e são aproximadamente normais. Também, o periodograma comprova que os resíduos apresentaram características de um ruído branco. Essas análises gráficas residuais forneceram o suporte necessário para o bom ajuste do modelo e, consequentemente, para a realização de inferências. Assim, a qualidade do modelo ajustado foi garantida pelas propriedades empíricas mostradas pelos resíduos.

Calculamos os RR e IC para cada modelo ajustado in loco e na região geral para cada poluente. Os RR estimados para um aumento de 10 na concentração de cada um dos poluentes atmosféricos para a região geral são apresentados na Tabela 2. Ao analisarmos os padrões apresentados nessa tabela, os gradientes evidenciaram claramente os efeitos significativos de $\mathrm{MP}_{10}$ nos atendimentos, para defasagens cumulativas. Para o $\mathrm{SO}_{2}$, na defasagem simples foram significativos os valores dos RR para o lag0 e o lag2. $\mathrm{O} \mathrm{NO}_{2}$, o $\mathrm{CO}$ e o $\mathrm{O}_{3}$ não apresentaram significância estatística no RR para a Região Metropolitana da Grande Vitória. 
Figura 2

Diagnóstico do modelo central para Região Metropolitana da Grande Vitória, Espírito Santo, Brasil.

2a) Contagem diária observada e prevista de atendimentos por doenças respiratórias

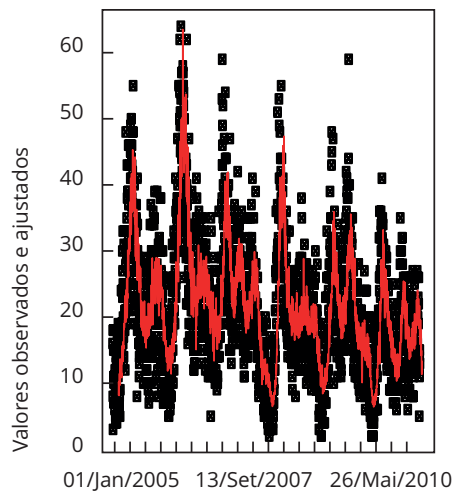

2d) PACF dos resíduos de ADR

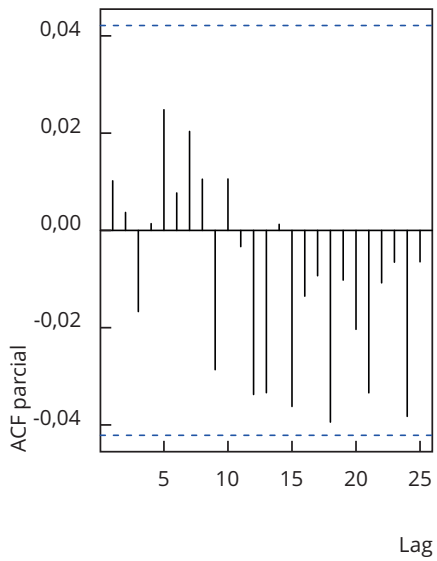

2b) Resíduos e séries de ADR

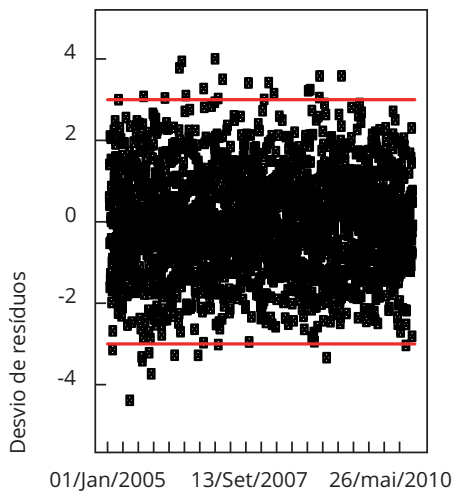

2e) Periodograma dos resíduos

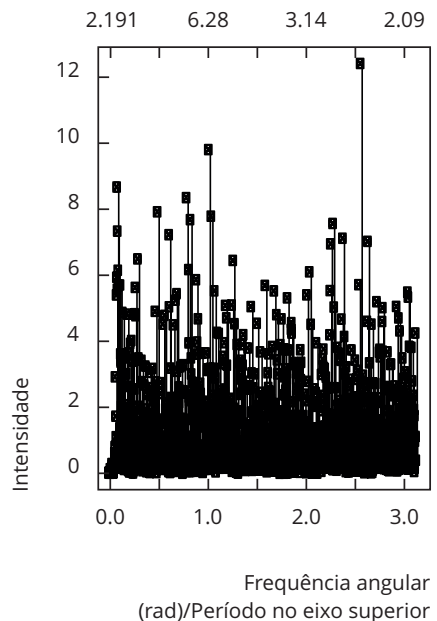

2c) Distância de Cook de observações de séries de ADR

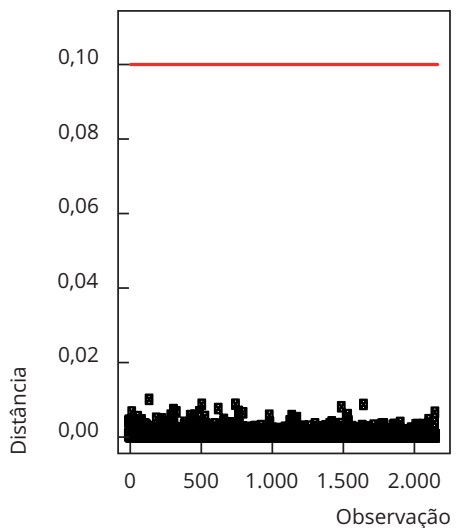

2f) Normalidade do gráfico de resíduos de ADR

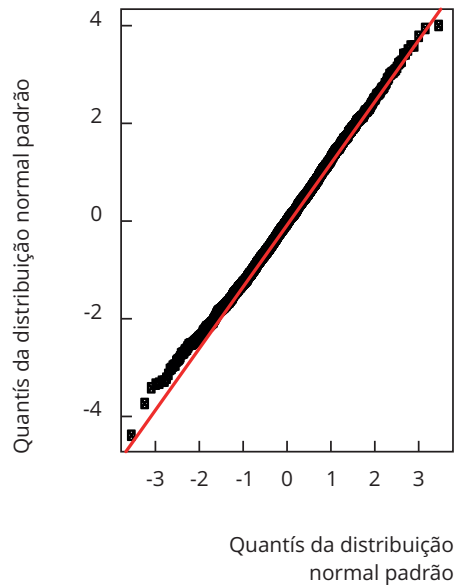

ADR: número diário de atendimentos por doenças respiratórias.

Nota: valores ajustados em vermelho.

$\mathrm{Na}$ Tabela 3, apresentamos um resumo dos valores de RR dos efeitos de cada poluente cujos cálculos apresentaram significância estatística, e os resultados que estão em negrito são os que apresentam maiores magnitudes. Verificamos que, para os poluentes $\mathrm{MP}_{10}$ e $\mathrm{SO}_{2}$, os valores estimados de RR foram maiores quando calculados para cada estação separadamente do que os obtidos para toda a Região Metropolitana da Grande Vitória, isto é, o modelo ajustado em que as covariáveis correspondem às médias das concentrações. Como visto anteriormente, os efeitos dos poluentes $\mathrm{NO}_{2}, \mathrm{CO}$ e $\mathrm{O}_{3}$ para a região agregada, Região Metropolitana da Grande Vitória, não apresentaram significância estatística no RR, o que pode ser considerado um resultado espúrio, pois os efeitos destes poluentes, para as localidades desagregadas por região, levaram a resultados de RR altamente significativos.

Toda análise foi pautada na comparação dos RR em relação aos diferentes poluentes e pontos espaciais, e comparados com uma média 


\section{Tabela 2}

Riscos relativos (RR) para atendimentos por doenças respiratórias em crianças menores de seis anos para um acréscimo de $10 \mu \mathrm{g} / \mathrm{m}^{3}$ de $\mathrm{MP}_{10}\left(\mu \mathrm{g} / \mathrm{m}^{3}\right), \mathrm{SO}_{2}\left(\mu \mathrm{g} / \mathrm{m}^{3}\right)$ e $\mathrm{NO}_{2}\left(\mu \mathrm{g} / \mathrm{m}^{3}\right), \mathrm{O}_{3}\left(\mu \mathrm{g} / \mathrm{m}^{3}\right)$ e $\mathrm{CO}\left(\mu \mathrm{g} / \mathrm{m}^{3}\right)$. Região Metropolitana da Grande Vitória, Espírito Santo, Brasil, 2005 a 2010.

\begin{tabular}{|c|c|c|c|c|}
\hline Exposição & $\% R R$ & Inferior & Superior & Valor de $p$ \\
\hline \multicolumn{5}{|l|}{$\mathrm{MP}_{10}$} \\
\hline Dia corrente & 0,99 & $-0,50$ & 2,50 & 0,19 \\
\hline Defasagem de 1 dia & 0,04 & $-1,35$ & 1,46 & 0,95 \\
\hline Defasagem de 2 dias & 0,81 & $-0,58$ & 2,23 & 0,25 \\
\hline Defasagem de 3 dias & 1,1 & $-0,29$ & 2,51 & 0,12 \\
\hline Acumulado de 2 dias & 0,69 & $-1,02$ & 2,42 & 0,43 \\
\hline Acumulado de 3 dias & 1,13 & $-0,82$ & 3,12 & 0,26 \\
\hline Acumulado de 4 dias & 1,77 & $-0,44$ & 4,03 & 0,12 \\
\hline Acumulado de 5 dias & 2,43 & $-0,05$ & 4,97 & 0,05 * \\
\hline Acumulado de 6 dias & 2,73 & 0,00 & 5,53 & 0,05 * \\
\hline Acumulado de 7 dias & 3,29 & 0,31 & 6,36 & 0,03 * \\
\hline Acumulado de 8 dias & 2,5 & $-0,67$ & 5,77 & 0,12 \\
\hline \multicolumn{5}{|l|}{$\mathrm{SO}_{2}$} \\
\hline Dia corrente & 4,47 & $-0,01$ & 9,14 & 0,05 * \\
\hline Defasagem de 1 dia & 4,20 & $-0,20$ & 8,79 & 0,06 \\
\hline Defasagem de 2 dias & 5,26 & 0,81 & 9,90 & 0,02 * \\
\hline Defasagem de 3 dias & 1,43 & $-2,89$ & 5,94 & 0,52 \\
\hline Acumulado de 2 dias & 6,47 & 0,99 & 12,26 & 0,02 * \\
\hline Acumulado de 3 dias & 8,80 & 2,55 & 15,44 & 0,01 * \\
\hline Acumulado de 4 dias & 8,76 & 1,93 & 16,05 & 0,01 * \\
\hline Acumulado de 5 dias & 7,09 & $-0,13$ & 14,84 & 0,05 * \\
\hline Acumulado de 6 dias & 3,70 & $-3,71$ & 11,69 & 0,34 \\
\hline Acumulado de 7 dias & 2,23 & $-5,45$ & 10,53 & 0,58 \\
\hline Acumulado de 8 dias & 0,00 & $-7,85$ & 8,52 & 1,00 \\
\hline \multicolumn{5}{|l|}{$\mathrm{NO}_{2}$} \\
\hline Dia corrente & 0,25 & $-0,89$ & 1,40 & 0,67 \\
\hline Defasagem de 1 dia & $-0,90$ & $-2,04$ & 0,25 & 0,12 \\
\hline Defasagem de 2 dias & $-0,45$ & $-1,56$ & 0,67 & 0,43 \\
\hline Defasagem de 3 dias & $-0,16$ & $-1,25$ & 0,94 & 0,78 \\
\hline Acumulado de 2 dias & $-0,50$ & $-1,90$ & 0,93 & 0,49 \\
\hline Acumulado de 3 dias & $-0,77$ & $-2,39$ & 0,88 & 0,36 \\
\hline Acumulado de 4 dias & $-0,82$ & $-2,63$ & 1,01 & 0,38 \\
\hline Acumulado de 5 dias & $-0,91$ & $-2,86$ & 1,09 & 0,37 \\
\hline Acumulado de 6 dias & $-0,91$ & $-3,02$ & 1,25 & 0,41 \\
\hline Acumulado de 7 dias & $-0,37$ & $-2,63$ & 1,95 & 0,75 \\
\hline Acumulado de 8 dias & $-0,31$ & $-2,72$ & 2,16 & 0,81 \\
\hline \multicolumn{5}{|l|}{$\mathrm{O}_{3}$} \\
\hline Dia corrente & 0,54 & $-0,54$ & 1,62 & 0,33 \\
\hline Defasagem de 1 dia & 0,46 & $-0,59$ & 1,52 & 0,39 \\
\hline Defasagem de 2 dias & 0,07 & $-0,97$ & 1,13 & 0,89 \\
\hline Defasagem de 3 dias & $-0,32$ & $-1,36$ & 0,73 & 0,55 \\
\hline Acumulado de 2 dias & 0,74 & $-0,55$ & 2,05 & 0,26 \\
\hline Acumulado de 3 dias & 0,69 & $-0,78$ & 2,19 & 0,36 \\
\hline Acumulado de 4 dias & 0,45 & $-1,20$ & 2,12 & 0,60 \\
\hline Acumulado de 5 dias & 0,34 & $-1,46$ & 2,17 & 0,71 \\
\hline Acumulado de 6 dias & 0,92 & $-1,04$ & 2,91 & 0,36 \\
\hline Acumulado de 7 dias & 0,66 & $-1,42$ & 2,78 & 0,54 \\
\hline Acumulado de 8 dias & 0,60 & $-1,60$ & 2,86 & 0,60 \\
\hline
\end{tabular}

(continua) 
Tabela 2 (continuação)

\begin{tabular}{lllll}
\hline Exposição & \%RR & Inferior & Superior & Valor de p \\
\hline CO & & & & \\
Dia corrente & 1,83 & $-3,25$ & 7,17 & 0,49 \\
Defasagem de 1 dia & $-3,61$ & $-8,21$ & 1,23 & 0,14 \\
Defasagem de 2 dias & 0,39 & $-4,37$ & 5,39 & 0,87 \\
Defasagem de 3 dias & 4,60 & $-0,26$ & 9,70 & 0,06 \\
Acumulado de 2 dias & $-1,69$ & $-7,76$ & 4,76 & 0,60 \\
Acumulado de 3 dias & $-1,24$ & $-8,30$ & 6,37 & 0,74 \\
Acumulado de 4 dias & 2,15 & $-5,97$ & 10,98 & 0,61 \\
Acumulado de 5 dias & 3,23 & $-5,74$ & 13,05 & 0,49 \\
Acumulado de 6 dias & 2,53 & $-7,11$ & 13,17 & 0,62 \\
Acumulado de 7 dias & 4,89 & $-5,72$ & 16,70 & 0,38 \\
Acumulado de 8 dias & 5,83 & $-5,60$ & 18,65 & 0,33 \\
\hline
\end{tabular}

Tabela 3

Aumento percentual e intervalo de 95\% de confiança (IC95\%) dos atendimentos pediátricos de emergência por sintomas respiratórios. Região Metropolitana da Grande Vitória, Espírito Santo, Brasil, 2005-2010.

\begin{tabular}{|c|c|c|c|c|}
\hline Exposição & $\% R R$ & IC95\% & Valor de $p$ & RAMQAr \\
\hline \multicolumn{5}{|l|}{$\mathrm{MP}_{10}$} \\
\hline Acumulado de 5 dias & 2,43 & $-0,05 ; 4,97$ & 0,05 & Região Metropolitana da Grande Vitória \\
\hline Acumulado de 6 dias & 2,73 & $-0,00 ; 5,53$ & 0,05 & Região Metropolitana da Grande Vitória \\
\hline Acumulado de 7 dias & 3,29 & 0,$31 ; 6,36$ & 0,03 & Região Metropolitana da Grande Vitória \\
\hline Defasagem de 1 dia & 4,49 & 1,$45 ; 7,62$ & 0,00 & Laranjeiras \\
\hline Acumulado de 2 dias & 4,5 & 1,$04 ; 8,08$ & 0,01 & Laranjeiras \\
\hline Acumulado de 3 dias & 5,17 & 1,$35 ; 9,13$ & 0,01 & Laranjeiras \\
\hline Acumulado de 4 dias & 4,66 & 0,$60 ; 8,89$ & 0,02 & Laranjeiras \\
\hline Defasagem de 1 dia & 4,66 & 0,$48 ; 9,02$ & 0,03 & Carapina \\
\hline Acumulado de 6 dias & 8,36 & 0,$16 ; 17,23$ & 0,05 & Carapina \\
\hline Acumulado de 7 dias & 12,27 & 3,$15 ; 22,19$ & 0,01 & Carapina \\
\hline Acumulado de 8 dias & 11,5 & 1,$85 ; 22,06$ & 0,02 & Carapina \\
\hline Acumulado de 6 dias & 10,59 & 1,$79 ; 20,16$ & 0,02 & Jardim Camburi \\
\hline Acumulado de 7 dias & 12,08 & 2,$63 ; 22,40$ & 0,01 & Jardim Camburi \\
\hline Acumulado de 8 dias & 11,82 & 1,$87 ; 22,73$ & 0,02 & Jardim Camburi \\
\hline Defasagem de 3 dias & 2,41 & 0,$17 ; 4,71$ & 0,03 & Enseada do Suá \\
\hline Dia corrente & 4,08 & 0,$54 ; 7,76$ & 0,02 & Vitória-Centro \\
\hline Acumulado de 6 dias & 6,58 & $-0,03 ; 13,64$ & 0,05 & Vitória-Centro \\
\hline Acumulado de 7 dias & 7,13 & 0,$01 ; 14,75$ & 0,05 & Vitória-Centro \\
\hline Acumulado de 7 dias & 9,05 & 1,$54 ; 17,12$ & 0,02 & Vila Velha-Centro \\
\hline Acumulado de 8 dias & 9,01 & 1,$19 ; 17,42$ & 0,02 & Vila Velha-Centro \\
\hline Defasagem de 3 dias & 2,62 & 0,$58 ; 4,71$ & 0,01 & Cariacica \\
\hline Acumulado de 7 dias & 4,53 & 0,$36 ; 8,88$ & 0,03 & Cariacica \\
\hline Acumulado de 8 dias & 4,77 & 0,$40 ; 9,34$ & 0,03 & Cariacica \\
\hline
\end{tabular}

(continua) 
Tabela 3 (continuação)

\begin{tabular}{|c|c|c|c|c|}
\hline Exposição & \%RR & IC95\% & Valor de $p$ & RAMQAr \\
\hline \multicolumn{5}{|l|}{$\mathrm{SO}_{2}$} \\
\hline Dia corrente & 4,47 & $-0,01 ; 9,14$ & 0,05 & Região Metropolitana da Grande Vitória \\
\hline Defasagem de 2 dias & 5,26 & 0,$81 ; 9,90$ & 0,02 & Região Metropolitana da Grande Vitória \\
\hline Acumulado de 2 dias & 6,47 & 0,$99 ; 12,26$ & 0,02 & Região Metropolitana da Grande Vitória \\
\hline Acumulado de 3 dias & 8,8 & 2,$55 ; 15,44$ & 0,01 & Região Metropolitana da Grande Vitória \\
\hline Acumulado de 4 dias & 8,76 & 1,$93 ; 16,05$ & 0,01 & Região Metropolitana da Grande Vitória \\
\hline Acumulado de 5 dias & 7,09 & 0,$13 ; 14,84$ & 0,05 & Região Metropolitana da Grande Vitória \\
\hline Dia corrente & 10,68 & 1,$09 ; 21,17$ & 0,03 & Laranjeiras \\
\hline Defasagem de 2 dias & 9,71 & 1,$68 ; 18,37$ & 0,02 & Jardim Camburi \\
\hline Acumulado de 7 dias & 13,52 & $-0,24 ; 29,18$ & 0,05 & Jardim Camburi \\
\hline Dia corrente & 8,7 & 1,$12 ; 16,85$ & 0,02 & Vila Velha-Centro \\
\hline Acumulado de 2 dias & 11,31 & 1,$23 ; 22,40$ & 0,03 & Vila Velha-Centro \\
\hline Defasagem de 3 dias & 11,9 & 0,$04 ; 25,17$ & 0,05 & Vila Velha-Centro \\
\hline Defasagem de 2 dias & 7,57 & 1,$17 ; 14,38$ & 0,02 & Cariacica \\
\hline \multicolumn{5}{|l|}{$\mathrm{NO}_{2}$} \\
\hline Defasagem de 2 dias & 3,85 & 0,$99 ; 6,80$ & 0,01 & Jardim Camburi \\
\hline Acumulado de 6 dias & 3,56 & 0,$35 ; 6,88$ & 0,03 & Vitória-Centro \\
\hline Acumulado de 7 dias & 3,88 & 0,$46 ; 7,42$ & 0,03 & Vitória-Centro \\
\hline Acumulado de 8 dias & 4,32 & 0,$70 ; 8,06$ & 0,02 & Vitória-Centro \\
\hline \multicolumn{5}{|l|}{$\mathrm{CO}$} \\
\hline Defasagem de 3 dias & 1,78 & $-0,02 ; 3,62$ & 0,05 & Laranjeiras \\
\hline Defasagem de 2 dias & 2,24 & 0,$72 ; 3,79$ & 0,00 & Ibes \\
\hline Defasagem de 3 dias & 1,97 & 0,$46 ; 3,51$ & 0,01 & Ibes \\
\hline Acumulado de 4 dias & 3,01 & 0,$60 ; 5,48$ & 0,01 & Ibes \\
\hline Acumulado de 5 dias & 2,96 & 0,$34 ; 5,65$ & 0,03 & Ibes \\
\hline Acumulado de 6 dias & 2,99 & 0,$20 ; 5,86$ & 0,04 & Ibes \\
\hline \multicolumn{5}{|l|}{$\mathrm{O}_{3}$} \\
\hline Dia corrente & 3,23 & 0,$17 ; 6,37$ & 0,04 & Laranjeiras \\
\hline Acumulado de 4 dias & 4,13 & $-0,08 ; 8,51$ & 0,05 & Laranjeiras \\
\hline
\end{tabular}

RAMQAr: Rede Automática de Monitoramento da Qualidade do Ar; RR: risco relativo.

\section{Discussão}

Neste estudo, nós analisamos o efeito da poluição atmosférica na saúde de crianças da Região Metropolitana da Grande Vitória por meio da estimativa do risco relativo em um MAG. As concentrações dos poluentes $\mathrm{MP}_{10}, \mathrm{SO}_{2}, \mathrm{NO}_{2}$ e $\mathrm{O}_{3}$, mesmo dentro dos padrões da legislação vigente e da OMS, mostraram associação significativa com o aumento do número de atendimentos em emergências por doenças respiratórias em crianças de 0 a 6 anos, o que comprova que não há limite de concentração de poluentes que seja seguro para a saúde humana 1.

O número desses atendimentos foi maior entre os meses de março a junho, que correspondem ao outono e início do inverno. Esse aumento esperado deve-se a diferentes fatores, como as baixas temperaturas que predispõem o agravamento de doenças respiratórias preexistentes, à maior incidência de doenças respiratórias virais e ao aumento da concentração dos poluentes primários determinada pela escassez de chuvas e ocorrência de inversão térmica.

Observamos que o maior número de crianças atendidas nas duas emergências residia na região da Enseada do Suá. Essa região sofre forte influência de fontes poluidoras móveis, pois está próxima a vias de grande tráfego automotor, incluindo uma ponte que liga o Município de Vitória ao de Vila Velha, por onde chegam a circular até 70 mil veículos por dia, e também de fontes fixas, representadas principalmente pelo complexo industrial localizado dentro da malha urbana. Um estudo recente 
mostrou que a população residente nessa região de Vitória é a que refere mais incomodo devido à poeira sedimentável 29 .

No período estudado, as concentrações dos poluentes variaram entre as diferentes estações da RAMQAr, o que pode ser justificado pelas atividades poluidoras específicas de cada região. As médias dos poluentes nessas estações são baseadas em dados in loco e são, em geral, bem próximas e diferenciadas de forma significativa da média geral. Essa evidência empírica mostra que as médias locais $\mathrm{E}\left(\mu \mathrm{L}_{t}\right),(\mathrm{L}=1, \ldots, 8)$ são diferentes da média regional $\mathrm{E}\left(\mu \mathrm{R}_{\mathrm{t}}\right)$. Esse é um resultado esperado, pois o processo não é estacionário nos momentos, isto é, $\mathrm{E}\left(\mu \mathrm{L}_{t}\right) \neq \mathrm{E}\left(\mu \mathrm{R}_{t}\right)$, para $\mathrm{L}=1, . ., 8$, e justifica o estudo proposto, no sentido de comparar o desfecho de saúde com relação aos poluentes nas áreas espacialmente discriminadas.

Os poluentes que mostraram associação mais consistente com o aumento do número de atendimentos por doenças respiratórias foram o $\mathrm{MP}_{10}$ e o $\mathrm{SO}_{2}$. O MP 10 apresentou um efeito substancial sobre os atendimentos em todas as defasagens e em quase todas as regiões analisadas, sendo observada maior magnitude nas regiões de Jardim Camburi e Carapina. Esse padrão de efeito está de acordo com o observado em outros estudos. Samoli et al. 10, na Grécia, observaram que um incremento de $10 \mu \mathrm{g} /$ $\mathrm{m}^{3}$ de $\mathrm{MP}_{10}$ e de $\mathrm{SO}_{2}$ estava associado com um aumento de 2,54\% e 5,98\% no número de hospitalizações por doenças respiratórias, respectivamente. Em Itabira, Minas Gerais, o aumento de $10 \mu \mathrm{g} /$ $\mathrm{m}^{3}$ na concentração de $\mathrm{MP}_{10}$ foi associado a um aumento de $4 \%$ nos atendimentos de crianças em pronto-socorro por doenças respiratórias para o lag0 e lag18. Na Região Metropolitana da Grande Vitória, Souza et al. 13 observaram relação significativa entre a concentração de todos os poluentes monitorados pela RAMQAr $\left(\mathrm{MP}_{10}, \mathrm{SO}_{2}, \mathrm{NO}_{2}, \mathrm{O}_{3}\right.$ e $\left.\mathrm{CO}\right)$ e o número de atendimentos em emergências devido a problemas respiratórios em crianças de até seis anos, por meio de uma modelagem híbrida, o modelo vetorial autorregressivo (VAR), a análise de componentes principais (ACP) e o MAG.

Observamos altas concentrações de $\mathrm{MP}_{10}$ na Estação de Cariacica, que podem ser justificadas pela localização da estação no Centro de Abastecimento do Espírito Santo (CEASA/ES), que além de ter um tráfego interno excessivo de veículos de grande porte, está próxima a duas vias de intenso tráfego de veículos pesados (BR-262 e BR-101).

A maior magnitude de efeito do $\mathrm{MP}_{10}$ e $\mathrm{SO}_{2}$, observada nas regiões de Jardim Camburi, Carapina e Laranjeiras, pode ser explicada pela proximidade destas regiões com vias de grande tráfego automor e com o maior polo industrial do Espírito Santo, que são fontes potenciais de $\mathrm{MP}_{10}$ e $\mathrm{SO}_{2}$.

Em relação ao $\mathrm{SO}_{2}$, observamos que os $\mathrm{RR}$ na área de abrangência das estações de Jardim Camburi e Laranjeiras foram os mais elevados. Na Enseada do Suá, o poluente que registrou média mais alta foi o $\mathrm{SO}_{2}$, entretanto, devido à falta de dados disponíveis, só foi calculado o $\mathrm{RR}$ para o $\mathrm{MP}_{10}$ nesta região, o que é uma falha deste estudo.

Os ajustes realizados no modelo, para cada uma das regiões no entorno das estações de monitoramento da RAMQAr, permitiram observar melhor o efeito de todos os poluentes sobre o número de atendimentos em emergências por doenças respiratórias. Existem evidências de que para estudos de séries temporais, medidas de uma estação central em comparação com modelos estatísticos ou de dispersão, ou estimativas derivadas de satélite, podem ser uma métrica de exposição adequada para poluentes distribuídos homogeneamente sobre a região estudada 30 , o que não se aplica ao presente trabalho.

Comparando as estimativas quando simulamos para toda a Região Metropolitana da Grande Vitória com as análises no entorno das estações da RAMQAr, alguns efeitos só foram percebidos quando as análises foram feitas nas localidades desagregadas por região, sinalizando um efeito de maior magnitude em relação à estimativa para a Região Metropolitana da Grande Vitória. A explicação para isso é que a análise pela média de todas as estações tende a suavizar os dados e assim diminuir a sua variabilidade, ocultando alguns efeitos e subestimando os efeitos da poluição do ar na saúde. Wilson et al. 31 também concluíram que considerar variações de concentração intraurbana em estudos epidemiológicos permite minimizar os erros de exposição e incertezas do risco relativo, no entanto, verificaram que esta consideração era atribuída somente a estudos de longo prazo. Entretanto, com essa metodologia observamos efeitos de curto prazo do $\mathrm{NO}_{2}$, do $\mathrm{CO}$ e do $\mathrm{O}_{3}$ sobre o número de atendimentos por doenças respiratórias, especialmente nas regiões de Jardim Camburi, Vila Velha-Centro e Laranjeiras. 
Apesar da associação entre os diversos poluentes e o risco de atendimento em emergências por doença respiratória em crianças na Região Metropolitana da Grande Vitória, observamos uma tendência decrescente dos níveis de poluentes ao longo do tempo, o que pode ser explicado pelo maior controle local da poluição do ar.

Para pesquisas futuras, outros grupos suscetíveis poderiam ser investigados na mesma região, para que seja possível elaborar um quadro completo dos efeitos agudos da poluição atmosférica na saúde da população. Como metodologia alternativa, técnicas de bootstrap poderão ser utilizadas com o objetivo de obter intervalos de mesma precisão, mas com menor amplitude amostral. Outra metodologia a ser considerada é a estimação da variância por meio de modelos heterocedásticos. Os modelos GLARMA 32 e PINAR 33, com maior complexidade estrutural, são ferramentas estatísticas que poderão ser abordadas nesses estudos.

A consistência das associações e a magnitude dos efeitos observados nas regiões analisadas, mesmo em um ambiente com níveis de poluentes dentro dos padrões estabelecidos pelas agências regulatórias, são extremamente relevantes em termos de saúde pública. Os resultados encontrados fornecem subsídios para a elaboração de medidas que visem a minimizar os riscos à saúde, contribuindo ainda com o planejamento de saúde ambiental e urbana e no aperfeiçoamento de políticas públicas.

\section{Colaboradores}

E. P. Matos participou da concepção e planejamento, coleta dos dados, análise e interpretação dos dados, elaboração do rascunho, redação final. V. A. Reisen, F. S. Serpa, P. R. Prezotti Filho e M. F. S. Leite colaboraram na concepção e planejamento, análise e interpretação dos dados, elaboração do rascunho, redação final.

\section{Informações adicionais}

ORCID: Emerson Pedreira Matos (0000-00024612-4784); Valdério Anselmo Reisen (0000-00028313-7648); Faradiba Sarquis Serpa (0000-00025703-4370); Paulo Roberto Prezotti Filho (00000002-9332-4121); Maria de Fátima Silva Leite (0000-0001-6445-1941).

\section{Agradecimentos}

Os autores agradecem o apoio dos órgãos de fomento à pesquisa: Conselho Nacional de Desenvolvimento Científico e Tecnológico (CNPq), Coordenação de Aperfeiçoamento de Pessoal de Nível Superior (Capes), Fundação de Amparo à Pesquisa do Espírito Santo (FAPES), Fundo de Apoio à Ciência e Tecnologia do Município de Vitória (FACITEC), Instituto Estadual de Meio Ambiente (IEMA), Hospital Infantil Nossa Senhora da Glória (HINSG) e Centro Integrado de Atenção à Saúde da Unimed Vitória (CIAS).

\section{Conflito de interesses}

Os autores declaram não haver conflito de interesses.

\section{Referências}

1. World Health Organization. Inheriting a sustainable world? Atlas on children's health and the environment. Geneva: World Health Organization; 2017.

2. Holgate ST. Every breath we take: the lifelong impact of air pollution - a call for action. Clin Med (Lond) 2017; 17:8-12.

3. Cifuentes LA, Vega J, Köpfer K, Lave LB. Effect of the fine fraction of particulate matter versus the coarse mass and other pollutants on daily mortality in Santiago, Chile. J Air Waste Manag Assoc 2000; 50:1287-98.

4. Gouveia N, Mendonça GAS, Ponce de Leon A, Correia JEM, Junger WL, Freitas CU, et al. Poluição do ar e efeitos na saúde nas populações de duas grandes metrópoles brasileiras. Epidemiol Serv Saúde 2003; 12:29-40.

5. Daumas RP, Mendonça GAS, Ponce de León A. Poluição do ar e mortalidade em idosos no município do Rio de Janeiro: análise de série temporal. Cad Saúde Pública 2004; 20:311-9.

6. Bakonyi SM, Danni-Oliveira IM, Martins LC, Braga AL. Poluição atmosférica e doenças respiratórias em crianças na Cidade de Curitiba - PR. Rev Saúde Pública 2004; 38:695-700.

7. Nascimento LF, Pereira LA, Braga AL, Módolo MCC, Carvalho Jr. JA. Effects of air pollution on children's health in a city in southeastern Brazil. Rev Saúde Pública 2006; 40:77-82.

8. Braga ALF, Pereira LAA, Procópio M, André PA, Saldiva PHN. Associação entre poluição atmosférica e doenças respiratórias e cardiovasculares na cidade de Itabira, Minas Gerais, Brasil. Cad Saúde Pública 2007; 23 Suppl 4:S570-8.

9. Chen R, Chu C, Tan J, Cao J, Song W, Xu X, et al. Ambient air pollution and hospital admission in Shanghai, China. J Hazard Mater 2010; 181:234-40. 
10. Samoli E, Nastos PT, Paliatsos AG, Katsouyanni K, Priftis KN. Acute effects of air pollution on pediatric asthma exacerbation: evidence of association and effect modification. Environ Res 2011; 111:418-24.

11. Almeida SP, Casimiro E, Calheiros J. Shortterm association between exposure to ozone and mortality in Oporto, Portugal. Environ Res 2011; 111:406-10.

12. Souza JB, Reisen VA, Santos JM, Franco GC. Principal components and generalized linear modeling in the correlation between hospital admissions and air pollution. Rev Saúde Pública 2014 ; 48:451-8.

13. Souza JB, Reisen VA, Franco GC, Ispány M, Bondon P, Santos JM. Generalized additive models with principal component analysis: an application to time series of respiratory disease and air pollution data. J R Stat Soc Series B Stat Methodol 2018; 67:453-80.

14. Nascimento AP, Santos JM, Mill JG, Souza JB, Reis Júnior NC, Reisen VA. Associação entre concentração de partículas finas na atmosfera e doenças respiratórias agudas em crianças. Rev Saúde Pública 2017; 51:3.

15. Froes Asmus CI, Camara VM, Landrigan PJ, Claudio L. A systematic review of children's environmental health in Brazil. Ann Glob Health 2016; 82:132-48.

16. Castro HA, Hacon S, Argento R, Junger WL, Mello CF, Castiglioni Júnior N. Air pollution and respiratory diseases in the Municipality of Vitória, Espírito Santo State, Brazil. Cad Saúde Pública 2007; 23 Suppl 4:S630-42.

17. Instituto Estadual de Meio Ambiente e Recursos Hídricos. Relatório da qualidade do ar: Grande Vitória 2013. Vitória: Instituto Estadual de Meio Ambiente e Recursos Hídricos; 2013.

18. Namdeo A, Tiwary A, Farrow E. Estimation of age-related vulnerability to air pollution: assessment of respiratory health at local scale. Environ Int 2011; 37:829-37.

19. Zeger SL, Thomas D, Dominici F, Samet JM, Schwartz J, Dockery D, et al. Exposure measurement error in time-series studies of air pollution: concepts and consequences. Environ Health Perspect 2000; 108:419-26.

20. Santolim L. Rede otimizada para o monitoramento da qualidade do ar da Grande Vitória, Espírito Santo [Dissertação de Mestrado]. Vitória: Universidade Federal do Espírito Santo; 1991.

21. Junger WL. Análise, imputação de dados e interfaces computacionais em estudos de séries temporais epidemiológicas [Tese de Doutorado]. Rio de Janeiro: Universidade do Estado do Rio de Janeiro; 2008.
22. Hastie TJ, Tibshirani RJ. Monographs on statistics and applied probability 43: generalizes additive models. New York: Chapman \& Hall; 1990.

23. Junger W, Leon AP. Ares: environment air pollution epidemiology. A library for time series analysis. Vienna: The Comprehensive $\mathrm{R} \mathrm{Ar}$ chive Network; 2011. (CRAN Archives).

24. Baxter LA, Finch SJ, Lipfert FW, Yu Q. Comparing estimates of the effects of air pollution on human mortality obtained using different regression methodologies. Risk Anal 1997; 17:273-8.

25. Zhang H, Niu Y, Yao Y, Chen R, Zhou X, Kan $H$. The impact of ambient air pollution on daily hospital visits for various respiratory diseases and the relevant medical expenditures in Shanghai, China. Int J Environ Res Public Health 2018; 15:E425.

26. Chen R, Yin P, Meng X, Liu C, Wang L, Xu X. Fine particulate air pollution and daily mortality. a nationwide analysis in 272 Chinese cities. Am J Respir Crit Care Med 2017; 196:73-81.

27. Katsouyanni K, Schwartz J, Spix C, Touloumi G, Zmirou D, Zanobetti A, et al. Short term effects of air pollution on health: a european approach using epidemiologic time series data: the APHEA protocol. J Epidemiol Community Health 1996; 50 Suppl 1:S12-8.

28. Qu Y, Pan Y, Niu H, He Y, Li M, Li L, et al. Short-term effects of fine particulate matter on non-accidental and circulatory diseases mortality: a time series study among the elder in Changchun. PLoS One 2018; 13:e0209793.

29. Melo MM, Santos JM, Frere S, Reisen VA, Reis Jr. NC, Leite MDFS. Annoyance caused by air pollution: a comparative study of two industrialized regions. International Journal of Environmental and Ecological Engineering 2015; 9:182-7.

30. Dionisio KL, Baxter LK, Burke LK, Özkaynak $\mathrm{H}$. The importance of the exposure metric in air pollution epidemiology studies: when does it matter, and why? Air Qual Atmos Health 2016; 9:495-502.

31. Wilson JG, Kingham S, Pearce J, Sturman AP. A review of intraurban variations in particulate air pollution: implications for epidemiological research. Atmos Environ 2005; 39:6444-62.

32. Davis RA, Dunsmuir WTM, Streett SB. Maximum likelihood estimation for an observation driven model for poisson counts. Methodol Comput Appl Probab 2005; 7:149-59.

33. Monteiro M, Scotto MG, Pereira I. Integervalued autoregressive processes with periodic structure. J Stat Plan Inference 2010; 140:1529-41. 


\section{Abstract}

The study aimed to investigate the short-term association between air pollution and emergency treatments for respiratory diseases in children 0 to 6 years of age. This was an ecological space-time study in Greater Metropolitan Vitória, Espirito Santo State, Brazil. A Poisson regression general additive model (GAM) used the number of daily treatments for respiratory diseases as the dependent variable, and the independent variables were daily concentrations of air pollutants $\left(\mathrm{PM}_{10} \mathrm{SO}_{2}\right.$ $\mathrm{NO}_{2}, \mathrm{O}_{3}$, and $\mathrm{CO}$ ), temperature, humidity, and precipitation. Average daily concentrations were used to make estimates for the entire metropolitan area and in loco analyses considering children residing in a $2 \mathrm{~km}$ radius around 8 air quality monitoring stations. An increase of $10 \mu \mathrm{g} / \mathrm{m}^{3}$ in the concentration of air pollutants increased the risk of emergency treatment for respiratory disease. In the overall area, for $P M_{10}$ the increase was $2.43 \%$, $2.73 \%$, and $3.29 \%$ in the cumulative values at 5 , 6 , and 7 days, respectively. For $\mathrm{SO}_{2}$, the increase was $4.47 \%$ on the day of exposure, $5.26 \%$ two days later, and $6.47 \%, 8.8 \%, 8.76 \%$, and $7.09 \%$ for the cumulative values at days $2,3,4$, and 5 days, respectively. CO showed a significant association for residents around two stations, and $\mathrm{O}_{3}$ for only one. Even within the limits set by the World Health Organization, the pollutants $\mathrm{PM}_{10} \mathrm{SO}_{2}$ $\mathrm{NO}_{2}$, and $\mathrm{O}_{3}$ are associated with increased risk of treatment for respiratory diseases in children 0 to 6 years of age, and some effects were only identified when disaggregating by neighborhood, i.e., in loco, which allows capturing greater variation in the data.

Air Pollution; Respiratory Tract Diseases; Child

\section{Resumen}

El objetivo fue investigar la asociación de corto plazo entre la contaminación del aire y la atención en urgencias por enfermedades respiratorias, en niños de 0 a 6 años. Estudio ecológico, espacial y temporal realizado en la Región Metropolitana de la Grande Vitória, Espírito Santo, Brasil. Se utilizó el modelo aditivo generalizado (MAG) de regresión de Poisson, con la variable dependiente que es el número diario de consultas por enfermedades respiratorias, y las variables independientes: concentraciones diarias de los contaminantes atmosféricos $\left(\mathrm{MP}_{10}, \mathrm{SO}_{2}, \mathrm{NO}_{2}, \mathrm{O}_{3}\right.$ y CO), temperatura, humedad y precipitación pluviométrica. Mediante las medias diarias de las concentraciones, se realizaron estimativas para toda la región y análisis in loco, considerando a niños residentes en un entorno de $2 \mathrm{~km}$ con 8 estaciones de monitoreo de la calidad del aire. El incremento de $10 \mu \mathrm{g} / \mathrm{m}^{3}$ en los niveles de concentración de los contaminantes atmosféricos aumentó el riesgo de atención en urgencias por enfermedad respiratoria. En la región como un todo, en el caso del $M P_{10}$ el aumento fue de 2,43\%, 2,73\% y 3,29\% en los acumulados de 5, 6 y 7 días, respectivamente. En el $\mathrm{SO}_{2}$ el incremento fue de 4,47\% durante el día de la exposición, 5,26\% dos días después, 6,47\%, 8, 8\%, 8,76\% y 7,09\% en los acumulados de 2, 3, 4 y 5 días, respectivamente. El CO presentó asociación significativa para residentes alrededor de dos estaciones, y el $\mathrm{O}_{3}$ solamente en una. Incluso dentro de los limites establecidos por la Organización Mundial de la Salud, los contaminantes $\mathrm{MP}_{10} \mathrm{SO}_{2}, \mathrm{NO}_{2}$ y $\mathrm{O}_{3}$ están asociados a un mayor riesgo en relación con la atención por enfermedades respiratorias en niños de 0 a 6 años, $y$ algunos efectos sólo se identificaron en las localidades desagregadas por región, esto es, in loco, lo que posibilita captar una mayor variabilidad de los datos.

Contaminación del Aire; Enfermedades

Respiratorias; Niño
Recebido em 24/Jul/2018

Versão final reapresentada em 20/Jan/2019

Aprovado em 05/Abr/2019 